\title{
Optimized QRT-PCR Approach for the Measurable Impact of Adjuvant Cholecalciferol Therapy in Ameliorating Cytokine Gene Expression
}

\author{
Javed Akram, Akram Tariq, Gibran Ali, Fridoon Jawad Ahmed, and Syeda Saba Aslam
}

\section{ABSTRACT}

The endemic Vitamin D deficiency in Pakistan and the current COVID-19 epidemic have converged into a double whammy scenario in Pakistan [1]. Nutritional epigenomic studies have highlighted Vitamin D as a master Vitamin influencing various genomic expressions through its active metabolite 1 $\alpha, 25$-dihydroxyvitamin D3 [2]. The objective of this study was to evaluate the measurable impact of adjuvant Cholecalciferol therapy in the Cytokine gene expression of COVID-19 patients by quantitative RealTime Polymerase Chain Reaction analysis. The trial was a randomized control prospective open label interventional trial done on moderate to severe COVID-19 patients with deranged inflammatory and coagulation biomarkers. SunnyD STAT (Vitamin D3 200000 IU) softgels were given at Day 1, Day 3 and Day 5 of the treatment. Optimized quantitative RealTime Polymerase Chain Reaction analysis showed decreased genetic expressions of Interleukin 6 (IL-6), Interleukin 2RA (IL-2RA) and Tumor Necrosis Factor (TNF-a) in the interventional group against the age and co-morbidities matched controls, providing molecular and genetic level evidence for the purported mechanism of amelioration of Cytokines induced pathogenic inflammation. However, inherent limitations of the design restrict the generalizability of the results and warrants caution for extrapolation. We recommend randomized placebo-controlled trials with larger sampling and genome wide profiling to infer more definite interpretations.

Keywords: Adjuvant therapy, Cholecalciferol, genetic expression, vitamin D3.

\section{INTRODUCTION}

Nutritional epigenomic studies have highlighted Vitamin $\mathrm{D}$ as a master Vitamin influencing various genomic expressions through its active metabolite $1 \alpha, 25$ dihydroxyvitamin D3 [2]. The activated Calcitriol binds to the nuclear Vitamin D Receptor (VDR), and confers its actions on thousands of loci within the human genome, consequently effecting the human epigenetics and transcriptome [2].

The pluripotent role of Vitamin D includes directly effecting the genetic expression of various inflammatory proteins, modulating the transcription factor activity and stimulating a cascade of intra cellular signaling [3]. Our study explores these interactions through genetic expressions as a possible molecular explanation for the early therapeutic intervention in the context of COVID-19.

\author{
Submitted: October 23, 2021 \\ Published: November 16, 2021 \\ ISSN: $2593-8339$ \\ DOI: $10.24018 /$ ejmed.2021.3.6.1117 \\ J. Akram \\ University of Health Sciences, Lahore, \\ Pakistan. \\ (e-mail: vc@uhs.edu.pk) \\ A. Tariq \\ Genomed Labortaries and Research \\ Center, Lahore, Pakistan. \\ (e-mail: akram@soe.ucsc.edu) \\ G. Ali \\ Okolohama State University, \\ Stillwater, OK, USA. \\ (e-mail: \\ lonsa25@student.london.ac.uk) \\ F. J. Ahmed \\ University of Health Sciences, Lahore, \\ Pakistan. \\ (e-mail: drfridoon@ ${ }^{\circledR}$ gmail.com) \\ S. S. Aslam* \\ Scotmann Pharmaceuticals, Islamabad, \\ Pakistan. \\ (e-mail: syedasabaaslam@gmail.com) \\ *Corresponding Author
}

\section{BACKGROUND}

The inert photosynthesized or dietary Vitamin D binds to the Gc globulin protein in the blood stream, the Vitamin D Binding Protein (DBP), and undergoes hepatic and renal hydroxylation for its conversion to its active metabolite $1,25(\mathrm{OH})_{2} \mathrm{D}$ [4]. Although the renal activation through CYP27B1 transcription is mainly dictated by the internal Calcium milieu of the body, an autocrine and paracrine fashion is followed through its high affinity attachment to the $(\mathrm{kD} 0.1 \mathrm{nM})$ ligand activated member of the transcription family of the nuclear receptors, the Vitamin D Receptor (VDR) in the extra renal tissues [5]. Consequently, heterodimerization of the VDR with Retinoid X Receptor (RXR) along with vitamin D response elements (VDREs) associated activation of the transcription factors is induced by this binding of $1,25(\mathrm{OH})_{2} \mathrm{D}$ with the VDRs [6]. The binding is specific to the response elements composed of $3 \mathrm{bp}$ (DR3 elements) separating the direct repeats of 5'-PuGG/TTCA-3'. Moreover, genetic repression with heterogeneous mechanisms is revealed though individual gene profiling 
studies requiring interactions with other classes of transcription factors also [8].

The activated Vitamin D Receptor binds to as many as nearly 20,000 loci within the chromatin, activating or repressing nearly $50 \%$ of the target genes it regulates, bringing about the genetic regulation of pluripotent physiological including the modulation of Cytokines gene expression in the immune system [9].

\section{A. Molecular VDR Influenced Genomic Activity}

The Vitamin D Receptor belongs to the Nuclear Receptors family, which are focused on various metabolic activities of the body [10]. Sensing the activated 1 $\alpha, 25$-dihydroxyvitamin D3, these Vitamin D Receptors express the genes in various tissues and cells of the human body, influencing their differentiation and epigenetic programming [11]. For instance, the binding of pioneer transcription factor, CCCTC binding factor or CTCF binding can be influenced by Vitamin $\mathrm{D}$ through VDR mediated binding to the less dense euchromatin through its genomic binding sites [12]. Moreover, Vitamin D can also influence the formation of Topologically Associated Domains (TADs), histone modifications and regulation of gene expression by modifying accessibility to Chromatin [13]. The Vitamin D Receptor, including its other family of nuclear receptors like RAR, PPAR, LXR, forms a heterodimer with retinoid X receptor [14]. This is evident through established VD signaling models. This complex prefers to bind to DR3-type response elements of the DNA where a repeat of 2 hexameric motifs is spaced by 3 nucleotides [15]. Moreover, this preferential motifs' binding of VDR to DR3-type sequences is confirmed through obtaining the VDR cistrome in a number of cell lines [16].

\section{B. Direct Epi-genomic Translational Effects of Vitamin D}

Interesting to highlight here is that the Vitamin D Receptor binds to only 200-2000 sites, depending on cell type, in the absence of a ligand [17]. However, the sites are increased by a factor of 2.5 after stimulation with the activated Calcitriol [18], marking the first direct epigenomic effect of Vitamin D [15]. The second effect is the modulation of nearly $10 \%$ of the binding sites of the cistrome subsets including the purinerich box 1 (PU.1), CEBPA and GABPA [19]. Moreover, nearly 1300 sites of the transcription factor CCCTC genome (CTCF) are also influenced by its activated form [20]. Another effect of $1,25[\mathrm{OH}]_{2} \mathrm{D}_{3}$, especially on THP-1 cells, is that it modulates the chromatin 3D structure through the Topologically Associated Domains (TADs) produced by the VDR sensitive CTCF [21]. Finally, $1,25[\mathrm{OH}]_{2} \mathrm{D}_{3}$ changes the chromatin accessibility by modifying the histone complex through the ligand-dependent interference of VDR [22] (Fig 1).

\section{Antagonization of Pro Inflammatory Transcription Factors by Vitamin D Receptor}

The main genomic role of Vitamin D in chronic inflammatory and autoimmune conditions involves the major histocompatibility complex and its co-stimulatory molecules including CD40, CD80 and CD86, which are repressed through their genetic codes by Vitamin D [23], [24]. Antagonization of pro inflammatory transcription factors by Vitamin D Receptor and its ligands leads to decreased

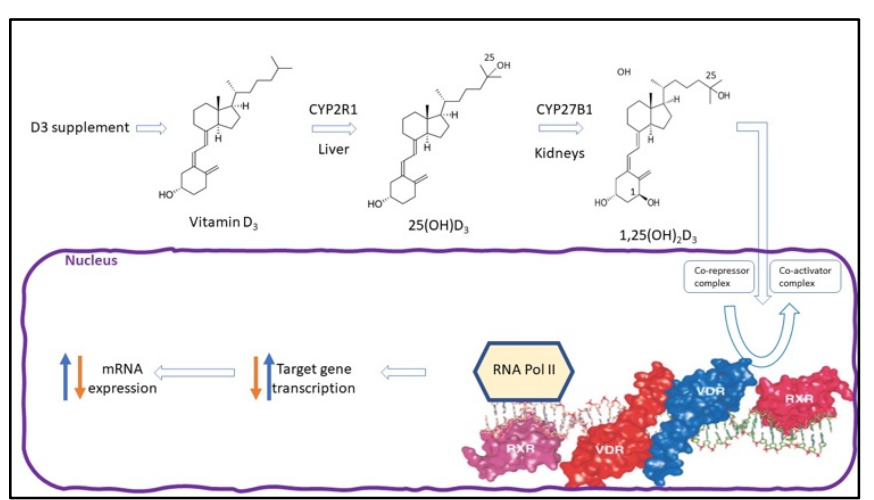

Fig. 1. Epi-genomic translational effects through transcriptome changes regulated by $1,25(0 \mathrm{H})_{2} \mathrm{D}$.

Cytokine expression of interleukins [25]. A study shortlisted 15 genes as the major targets of Vitamin D in the immune system, majority of which were found to encode proteins present in or on the cellular membranes, including SEMA6B, TREM1, LILRB4, NINJ1, CD14, THBD, CD93, LRRC25, ACVRL1 [26].

\section{Impact on Cytokine Gene Expression of IL-6, IL-2 and TNF- $a$}

Since pro-inflammatory Cytokines like Interleukins-6, Interleukins-2 and Tumor Necrosis Factor-alpha are associated with the pathogenic inflammation in COVID-19, a study done on COVID-19 patients at Mont Sinai health system, New York, predicted the COVID-19 severity and survival through identifying inflammatory cytokine signatures, serving as predictive bio markers of pathogenic inflammation and impending Cytokine storm [27]. These signatures helped in guiding targetable immune pathways. Studies exploring applicability of this guide could be important. Moreover, the practical application of the use of Cytokine signature expressions guide is also lacking, especially in the context of $1,25(\mathrm{OH}) \mathrm{D}$. Therefore, the objective of this randomized control trial was to identify the genetic level impact of loading Cholecalciferol adjuvant therapy (200000 IU on day 1, 3 and 5) on the Cytokine inflammatory signatures, by following its molecular pathway.

\section{MATERIALS AND Methods}

\section{A. RNA Isolation and Real-Time PCR Analysis for IL-2, IL- 6 and TNF- $a$}

Sample was collected in anti-coagulant Ethylenediaminetetraacetic acid [EDTA] tubes $1.5 \mathrm{ml}$ and 2 $\mathrm{ml}$ [Eppendorf]. Nano-drops [RNA later Thermo Fisher Scientific] confirmed total RNA integrity. Cytokines Genetic expressions were done using peripheral blood mononuclear cells [PBMCs] through the direct binding asymmetrical Cyanine SYBR Green dye and Superscript III Platinum One Step Quantitative Real Time - Polymerase Chain Reaction kit.

Assessment of the mRNA genes expression of IL-6, IL2 and TNF-a was done with appropriate primers and probes. RT-PCR primer/Probe Sets by Applied Biosystems were used for the inflammatory and coagulation markers. Superscript III Platinum One Step Quantitative Real Time Polymerase Chain Reaction kit [Thermo fisher], QI Aamp 
Viral RNA Mini Kit [Qiagen Inc USA] and Phusion U Green Multiplex Polymerase Chain Reaction Master Mix and RNA later [Thermo Scientific ${ }^{\mathrm{TM}}$ ] were in use. Polymerase Chain Reaction amplification conclusion was made following 3 cycles comprising of the first warm up cycle for 15 seconds at $95{ }^{\circ} \mathrm{C}$, second denaturation cycle at $56{ }^{\circ} \mathrm{C}$ for 30 seconds and finally 30 seconds at $72{ }^{\circ} \mathrm{C}$ acquiring Target SYBR Green.

\section{B. Study Design and Ethical Approvals}

The study design was a prospective open labeled randomized interventional trial executed at Akram Medical Complex, Lahore and Jinnah hospital, Lahore in collaboration with University of Health Sciences, Lahore after receiving the ethical approvals by the Ethical Review Committee [ERC] of the University of Health Sciences, Lahore [rc no. UHS/REG-20/ERC/1762 dated 8 September 2020] and National Bioethical Committee [ref. no. 487/NBC-COVID-50/20/699 dated November 19,2020] and Clinical Trial Unit of the Drug Regulatory Authority of Pakistan [IN-1417/SN-2037470-1/CTU-DRAP dated November 26,2020]. Written informed consent was taken from all subjects.

\section{Sampling}

Classical markers like Ferritin, D-Dimers, CRP and LDH were used to stratify patients based on inflammation and disease severity for inclusion. Confirmed moderate to severe COVID-19 patients, age-matched 40-70 years with comorbidities including Diabetes and cardiac disease, DDimers $>500 \mathrm{ng} / \mathrm{ml}, \mathrm{CRP}>10 \mathrm{mg} / \mathrm{L}, \mathrm{LDH}=125-243 \mathrm{U} / \mathrm{L}$, Ferritin 20 to $500 \mathrm{ng} / \mathrm{ml}$ for males and 20 to $200 \mathrm{ng} / \mathrm{ml}$ for females were included while those with respiratory failure, shock, pregnancy, lactation or multiple organ damage were excluded. Openepi.com was used to calculate the sample size. Two-sided significance level (1-alpha) was taken as $95 \%$ and power of $80 \%$ with sample size ratio as 1 . Sample size for control and interventional group, was calculated as 11 each (total 22). A total sample size of 26 was derived by Fleiss with Continuity Correction. For the purpose of better analysis and to overcome bias of non-responder's or open labeling, we doubled the sample size $(\mathrm{n}=2 \times$ sample size $)$, and so 52 patients were recruited for clearer outcomes. With a missing or inadequate data, 2 samples were dropped and analysis was done on 50 samples. Baseline characteristics of the samples is given in Table I.

TABLE I: BASELINE CHARACTERISTICS OF PARTICIPANTS N=50

\begin{tabular}{ccc}
\hline \hline \multicolumn{2}{c}{ TABLE I: BASELINE CHARACTERISTICS OF PARTICIPANTS N=50 } \\
\hline \multirow{2}{*}{ Gender n (\%) } & Male n (\%) & $20(40 \%)$ \\
& Female n (\%) & $30(60 \%)$ \\
\hline \multirow{2}{*}{ ZAge n (\%) } & Mean + SD & $54.7 \pm 14.79$ \\
& Median & 56 \\
& Mode & 60 \\
\hline \multirow{2}{*}{ Co-morbidities } & Diabetes & $21(42 \%)$ \\
n (\%) & Hypertension & $29(58 \%)$ \\
& History of Stroke/CVD & $3(6 \%)$ \\
\hline \multirow{2}{*}{ Lab markers } & D-Dimers (ng/ml) & $1090+460$ \\
(Mean + SD) & LDH (U/L) & $460+35$ \\
& CRP (mg/L) & $29+8$ \\
\hline \hline
\end{tabular}

\section{Lab Screening}

After admission in the COVID-19 ward, moderate to severe patients were screened for their serum 25(OH)D3 levels, inflammatory and coagulation bio markers including D-Dimer, Ferritin, CRP and LDH.

Direct chemiluminometric two-site sandwich immunoassay method and direct chemiluminometric one step immunoassay method were used to estimate Ferritin and Interleukin 6 [IL6] respectively, on Advia Centaur XPT machine [Siemens Healthineers, Erlangen, Germany].

Photometric kinetic UV-IFCC and photometric immunoturbimetric methods were used to estimate Lactate dehydrogenase $[\mathrm{LDH}]$ and $\mathrm{C}$ reactive protein [CRP] respectively using AU5800 Beckman Coulter machine. Serum D levels were analyzed through Enzyme Linked Immunoassay (ELISA) kit Abcam, USA.

\section{E. Randomization}

COVID-19 positive patients with serum Calcitriol levels below $30 \mathrm{ng} / \mathrm{ml}$ and having the abnormal inflammatory and coagulation biomarkers as defined in the inclusion criteria were randomized into two groups namely the interventional group/Adjuvant SunnyD STAT softgel group (VD group) and control group (usual treatment only). Computer generated randomization according to patient's serial number was followed. Patients in the SunnyD group received adjuvant SunnyD STAT softgel (200000 IU Vitamin D) on the first, third and fifth day of their usual COVID-19 treatment. The control group received usual COVID-19 treatment according to institutional protocols. Repeat testing of serum inflammatory and coagulation biomarkers was done on day 3 and day 5, while multiplex real time monitoring of Cytokine expressions was observed.

\section{F. Rapid Multiplex Cytokine Assay and Statistical Analysis}

A rapid multiplex Cytokine assay was implemented to measure serum IL-6, tumor necrosis factor (TNF- $\alpha$ ) and IL2RA in the included cases and controls. Results from both sub groups underwent analysis and comparison. For the differences in the serum bio markers and cytokine expressions. Statistical analysis was done using SPSS [version 25].

Baseline characteristics of participants were presented using standard descriptive statistics including mean + standard deviation, median and mode for continuous variables and categorical variables were displayed by count $[\mathrm{n}]$ and percentages $(\%)$.

For the analysis of significance, one-way analysis of variance (ANOVA), followed by post-hoc Tukey's test were used. Within group comparison was done by paired t-test, with data expression in mean + SD format for normal numerical. $\mathrm{P}$ value below 0.05 was considered significant. Fold change expression of each gene was calculated.

\section{RESUlTS}

Fold change in the gene expression of the molecular inflammatory markers including IL-6, IL-2RA and TNF- $\alpha$ was calculated. Down regulation of $>4$-fold, $>2.5$-fold and $>2$-fold was observed in the molecular gene expression of Tumor Necrosis Factor (TNF-a), Interleukin-6 (IL-6) and 
Interleukin Receptor Alpha IL-2RA, respectively (Fig. 2, 3, 4).

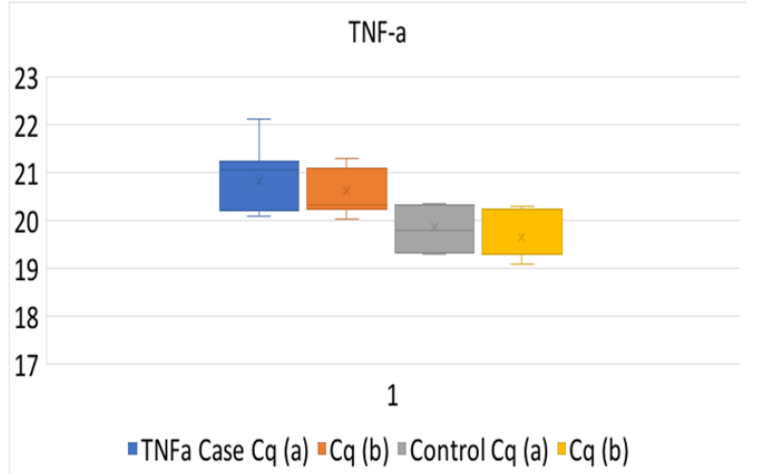

Fig. 2. Fold change in the gene expression of Tumor Necrosis Factor Alpha (TNF-a): where $\mathrm{Cq}=$ Quantification Cycle $\mathrm{a}=$ before intervention $\mathrm{b}=\mathrm{after}$ intervention.

\section{IL-6}

36

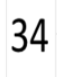

32

30

28

26

1

"IL6 Case Cq (a) "Cq (b) "Control Cq (a) "Cq (b)

Fig. 3. Fold change in the gene expression of Interleukin-6 (IL-6); where $\mathrm{Cq}=$ Quantification Cycle $\mathrm{a}=$ before intervention $\mathrm{b}=$ after intervention.

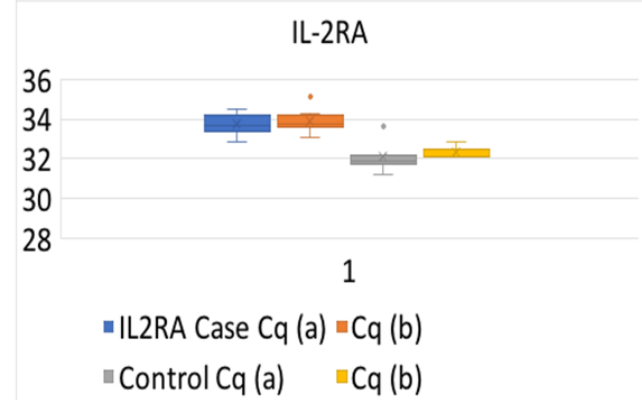

Fig. 4. Fold change in the gene expression of Interleukin Receptor Alpha (IL 2RA) where $\mathrm{Cq}=$ Quantification Cycle $\mathrm{a}=$ before intervention $\mathrm{b}=$ after intervention.

A p-value of $<0.05$ was considered to be statistically significant. With this, the comparative analysis through paired t-test showed statistically significant $(\mathrm{p}<0.0001)$ reduction in the Cytokine genetic expression level as calculated for the interventional group, while the results were not so significant $(\mathrm{p}>0.05)$ for the age and comorbiditiesmatched controls using the quantification Cycle $(\mathrm{Cq})$ indicating the amplification curve with respect to the cycle axis. The difference in expression of Cytokines between the groups (interventional versus control) was significant at $\mathrm{p}<0.05$, with the expression being markedly high in the control group as compared to the interventional group (Table II).

One-way variance analysis (ANOVA) for each genetic expression variable was carried out. For Interleukin-6 (IL-6), a mean of $33.7571 \pm 0.7413$ was calculated for the Cases, while the controls showed a mean of $31.705 \pm 1.1276$ and the f-ratio value of 61.728 . The p-value was statistically significant at $<0.00001$. For Interleukin Receptor Alpha (IL2RA), the mean value was $33.9156 \pm 0.5144$, while for the controls it was $32.3336 \pm 0.269$ the f-ratio value was calculated to be 185.68975 . and the p-value was significant at $<0.00001$. For Tumor Necrosis Factor (TNF-a), the mean was $20.6444 \pm 0.5009$ for the cases and $19.6776 \pm 0.4754$ for the controls with the f-ratio value of 49.0012 . The $p$-value was significant as it was $<0.00001$ (Table III).

TABLE II: COMPARATIVE ANALYSIS THROUGH PAIRED T-TEST

\begin{tabular}{|c|c|c|c|c|c|c|c|}
\hline & \multicolumn{2}{|c|}{ Group } & \multirow[b]{2}{*}{$\begin{array}{c}\text { Two-tailed } \mathrm{p} \\
\text { value }\end{array}$} & \multirow[b]{2}{*}{$95 \%$ CI } & \multirow[b]{2}{*}{$\mathrm{t}$} & \multirow[b]{2}{*}{ Df } & \multirow[b]{2}{*}{ SE of diff } \\
\hline & Cases $(n=25)$ & Controls $(n=25)$ & & & & & \\
\hline \multicolumn{8}{|c|}{ TNF $-\alpha$} \\
\hline$\underset{\text { SEM }}{\text { Mean }}+$ SD & $\begin{array}{c}20.6444 \pm 0.5009 \\
0.1002\end{array}$ & $\begin{array}{c}19.6776 \pm 0.4754 \\
0.0951\end{array}$ & $<0.0001$ & $0.6406-1.2930$ & 6.116 & 24 & 0.158 \\
\hline \multicolumn{8}{|c|}{ IL-6 } \\
\hline$\underset{\text { SEM }}{\text { Mean }}+$ SD & $\begin{array}{c}33.7660 \pm 0.7545 \\
0.1509\end{array}$ & $\begin{array}{c}31.6428 \pm 0.2294 \\
0.2294\end{array}$ & $<0.0001$ & $1.4926-2.7538$ & 6.949 & 24 & 0.306 \\
\hline \multicolumn{8}{|c|}{ IL-2RA } \\
\hline $\begin{array}{c}\text { Mean }+ \text { SD } \\
\text { SEM }\end{array}$ & $\begin{array}{c}33.9464+0.5039 \\
0.1008\end{array}$ & $\begin{array}{c}32.3336+0.2690 \\
0.0538\end{array}$ & $<0.0001$ & $1.4026-1.8230$ & 15.84 & 24 & 0.102 \\
\hline
\end{tabular}

TABLE III: ANOVA ONE WAY TEST

\begin{tabular}{|c|c|c|c|c|}
\hline & Cases & Control & Total & \\
\hline $\mathrm{N}$ & 28 & 24 & 52 & \\
\hline Mean \pm SD & $33.7571 \pm 0.7413$ & $31.705 \pm 1.1276$ & $32.81 \pm 1.3898$ & \\
\hline$\Sigma X$ & 945.2 & 760.92 & 1706.12 & \\
\hline$\Sigma X 2$ & 31922.0888 & 24154.21 & 56076.3 & \\
\hline \multicolumn{5}{|c|}{ Results } \\
\hline Source & $S S$ & $d f$ & $M S$ & \\
\hline Between groups & 54.4228 & 1 & 54.4228 & $F=61.728$ \\
\hline
\end{tabular}




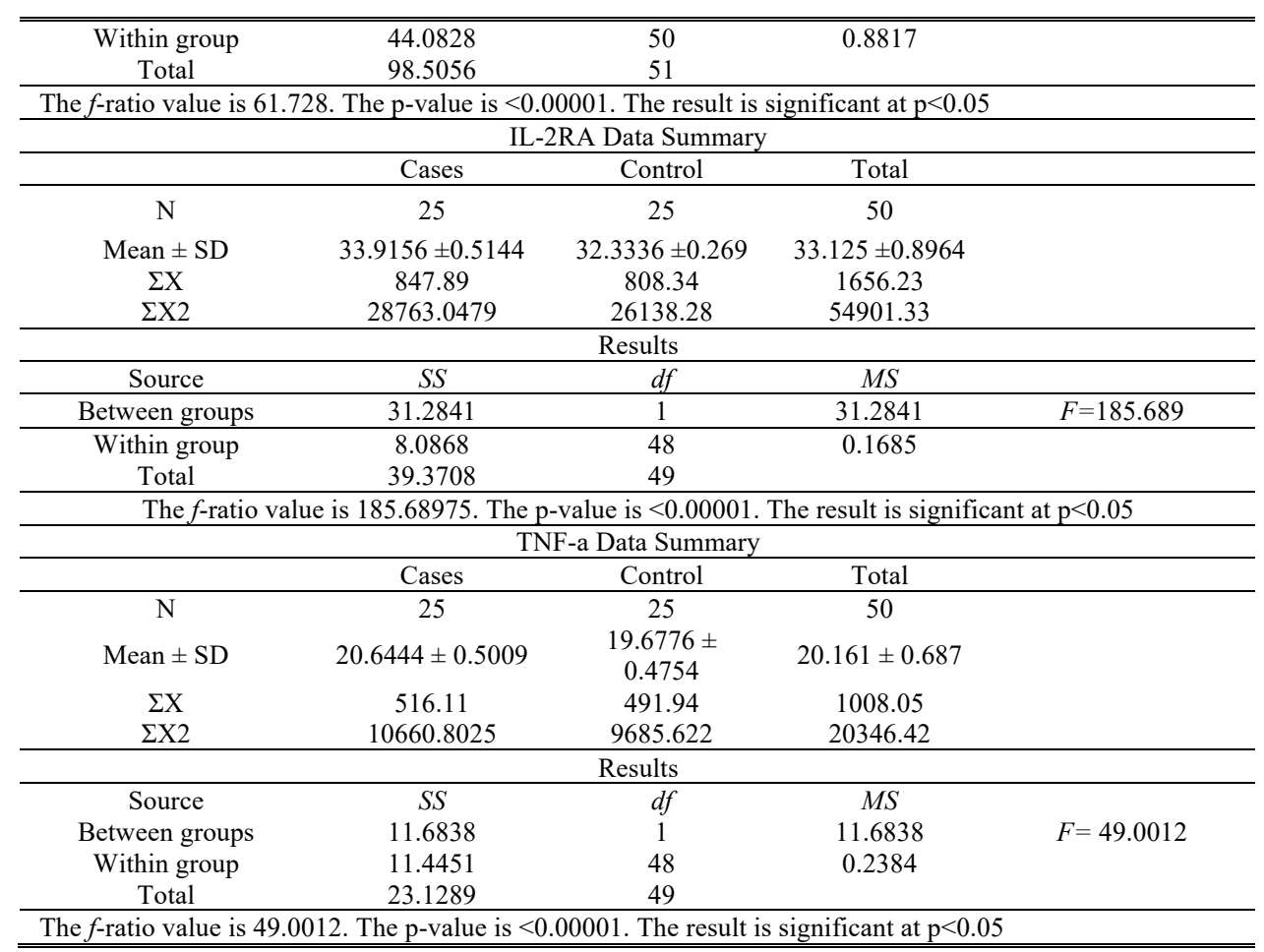

\section{DISCUSSION}

Although the two groups under randomization (controls and interventional) were matched with respect to their comorbidities, age, vital parameters, symptoms intensity and duration, significant difference in the interventional and control groups after the intervention were intriguing.

Rapid increase in the serum Vitamin D3 levels after oral ingestion of softgel within a short period of time showed that the pharmacodynamics of softgel delivery system in raising serum $25(\mathrm{OH}) \mathrm{D} 3$ are favorable, when results are required urgently.

The rapid and significant decrease in the coagulation and bio inflammatory markers after the adjuvant therapy of Vitamin D, point toward the immunomodulatory potential of Vitamin D in COVID-19 and early identification and treatment can help in halting the impeding Cytokine storm the main perpetrator in COVID-19 related morbidities and mortalities.

To the best of our knowledge, this study is the first randomized control trial to evaluate the applicability of the predictive guide of the Cytokine genes signature expression, through adjunctive therapy of an immunomodulator in the form of Vitamin D3.

Thus, our results show promising role of loading doses of Vitamin D3 in the initial phases of COVID-19, modulated through the repercussions of a controlled immune response, to prevent the blistering pace entry into the immunological apocalypse.

The rationale for using the selected inflammatory bio markers panel was based on existing literature. Studies have labelled Interleukins- 6 as one of the most important and independent bio markers for COVID-19 prognosis with respect to mortality, survival, use of ventilation and end organ damage even more than the prognostic prediction offered by C-Reactive proteins and D-dimer levels, after adjustment with other potentially influencing factors like socio- demographic features and presence/absence of comorbidities [28]-[33], [40]. Also, TNF- $\alpha$ has been highlighted as a strong predictor with increased levels associated with end organ damage and poor outcome after adjustment [34], [35], [40]. We also included Interleukin-2RA in our assessment panel of Cytokines because it has also been showing an independent association with survival time, after adjusting with all other co variables, in various studies [36]-[40].

Since pro inflammatory Cytokines like interleukins-6, interleukins-2 and Tumor Necrosis Factor-alpha are associated with the pathogenic inflammation in COVID-19, a study done on COVID-19 patients at Mont Sinai health system, New York, predicted the COVID-19 severity and survival through identifying inflammatory cytokine signatures, serving as predictive bio markers of pathogenic inflammation and impending Cytokine storm [40].

These signatures helped in guiding targetable immune pathways. Studies exploring applicability of this guide could be important. Therefore, we aimed to explore the molecular level impact of Vitamin D supplementation on the genetic expression of these pro inflammatory markers and to see the association if any with the serum 25[OH]D level.

Moreover, the practical application of the use of Cytokine signature expressions guide is also lacking. Therefore, the greater objective of this trial was to halt the impending danger of a Cytokine storm through identifying Cytokine inflammatory signatures at the genetic level and adding SunnyD STAT softgel (Vitamin D3 200000 IU) as an adjuvant therapy on day 1,3 and 5 of patient enrollment.

Notably, the cytokine response of the interventional group showed statistically significant difference from the traditional treatment control group, which sustained elevated cytokine levels over the study period. This raises the possibility of a mitigation strategy with immune modulatory role of Vitamin $\mathrm{D}$ and the window of opportunity for its adjunctive use. This study might add to the guides for such adjunctive therapies based on mechanistic association and modulation of genetic 
expression of cytokines, providing a rational approach for future strategies.

\section{StRengths AND Limitations}

Due to the strict criteria for inclusion, age matching and molecular level real time multiplex analysis through RTPCR, sample size could not be very large. Therefore, generalizability of the results should be extrapolated with caution. Additionally, the inherent limitations of an open labelled trial could be present. Future randomized placebo control large trials are warranted to infer more definite interpretations. However, to the best of our knowledge, this was the first randomized control trial, especially in this region, evaluating the adjuvant immune modulatory role of Vitamin D supplementation in the moderate-severe COVID19 patients' cohort, providing molecular and genetic level evidence for the purported mechanism of amelioration of Cytokines induced pathogenic inflammation.

\section{CONCLUSION}

Genetic expression profiling of the Cytokines showed improvements after the addition of Cholecalciferol adjuvant therapy to the COVID-19 treatment regime, providing molecular and genetic level evidence for the purported mechanism of amelioration of Cytokines induced pathogenic inflammation. However, inherent limitations of the design restrict the generalizability of the results and warrants caution for extrapolation. We recommend randomized placebocontrolled trials with larger sampling and genome wide profiling to infer more definite interpretations.

\section{ACKNOWLEDGEMENTS}

We acknowledge Dr. Shehla Akram for providing and arranging patients at her Akram Medical Complex, Lahore and all those who were involved in the logistics and data collection process.

\section{NOMENCLATURE}

The general nomenclature used for this trial during inception, designing and execution was the VICCTORI trial denoting "Vitamin D In Corona Cytokine Therapy on Registered Inpatients".

\section{REFERENCES}

[1] Farooka, Waris M, Aslam SS, Tarar MA, Rafi M, Ahmad A, Rafique N, Bilal M. Vitamin D perspective in front line healthcare workers amid COVID-19. European Journal of Medical and Health Sciences. 2021; 3(4): $65-72$.

[2] Carlberg C. Nutrigenomics of vitamin D. Nutrients. 2019; 11(3): 676.

[3] Ghareghomi S, Ahmadian S, Zarghami N, Kahroba H. Fundamental insights into the interaction between telomerase/TERT and intracellular signaling pathways. Biochimie. 2020.

[4] Bikle DD. Vitamin D biochemistry and physiology. In Extraskeletal Effects of Vitamin D. Humana Press, Cham. 2018; 1-40.

[5] Zhu M, Tan Z, Luo Z, Hu H, Wu T, Fang S, et al. Association of the vitamin D metabolism gene GC and CYP27B1 polymorphisms with cancer susceptibility: a meta-analysis and trial sequential analysis. Bioscience Reports. 2019; 39(9): BSR20190368.
[6] Staniszewski LJ, Shahani P, Heck M, Hasan DS, Wagner C, Jurutka $\mathrm{PW}$. Assessment of novel vitamin d receptor antagonists that mediate suppression of vitamin D signaling. The FASEB Journal. 2018; 32: $1 \mathrm{~b} 98$.

[7] Dimitrov V, Barbier C, Ismailova A, Wang Y, Dmowski K, SalehiTabar R, et al. Vitamin D-regulated gene expression profiles: Speciesspecificity and cell-specific effects on metabolism and immunity. Endocrinology. 2021; 162(2): bqaa218.

[8] Al-Daghri NM, Torretta E, Capitanio D, Fania C, Guerini FR, Sabico $\mathrm{SB}$, et al. Intermediate and low abundant protein analysis of vitamin D deficient obese and non-obese subjects by MALDI-profiling. Scientific Reports. 2017; 7(1): 1-10.

[9] Carlberg C. Vitamin D genomics: from in vitro to in vivo. Frontiers in endocrinology. 2018; 9: 250.

[10] Zenata O, Vrzal R. Fine tuning of vitamin D receptor (VDR) activity by post-transcriptional and post-translational modifications. Oncotarget. 2017; 8(21): 35390.

[11] Meza-Meza MR, Ruiz-Ballesteros AI, de la Cruz-Mosso U. Functional effects of vitamin D: From nutrient to immunomodulator. Critical Reviews in Food Science and Nutrition. 2020: 1-21.

[12] Carlberg C. Molecular endocrinology of vitamin D on the epigenome level. Molecular and Cellular Endocrinology. 2017; 453: 14-21.

[13] Perino M, Veenstra GJ. Chromatin control of developmental dynamics and plasticity. Developmental Cell. 2016; 38(6): 610-620.

[14] Mangelsdorf DJ, Evans RM. The RXR heterodimers and orphan receptors. Cell. 1995; 83(6): 841-50.

[15] Carlberg C, Bendik I, Wyss A, Meier E, Sturzenbecker LJ, Grippo JF, et al. Two nuclear signalling pathways for vitamin D. Nature. 1993; 361(6413): 657-660.

[16] Carlberg C. Genome-wide (over) view on the actions of vitamin D. Frontiers in Physiology. 2014; 5: 167.

[17] Deeb KK, Trump DL, Johnson CS. Vitamin D signalling pathways in cancer: potential for anticancer therapeutics. Nature Reviews Cancer. 2007; 7(9): 684-700.

[18] Tuoresmäki P, Väisänen S, Neme A, Heikkinen S, Carlberg C. Patterns of genome-wide VDR locations. PloS One. 2014; 9(4): e96105.

[19] Seuter S, Neme A, Carlberg C. Epigenomic PU. 1-VDR crosstalk modulates vitamin D signaling. Biochimica et Biophysica Acta (BBA)Gene Regulatory Mechanisms. 2017; 1860(4): 405-415.

[20] Neme A, Seuter S, Carlberg C. Vitamin D-dependent chromatin association of CTCF in human monocytes. Biochimica et Biophysica Acta (BBA)-Gene Regulatory Mechanisms. 2016; 1859(11): 13801388.

[21] Carlberg C, Neme A. Machine learning approaches infer vitamin D signaling: Critical impact of vitamin D receptor binding within topologically associated domains. The Journal of Steroid Biochemistry and Molecular Biology. 2019; 185: 103-109.

[22] Dunlop TW, Väisänen S, Frank C, Molnár F, Sinkkonen L, Carlberg C. The human peroxisome proliferator-activated receptor $\delta$ gene is a primary target of $1 \alpha, 25$-dihydroxyvitamin D3 and its nuclear receptor. Journal of Molecular Biology. 2005; 349(2): 248-260.

[23] Barragan M, Good M, Kolls JK. Regulation of dendritic cell function by vitamin D. Nutrients. 2015; 7(9): 8127-8151.

[24] Limketkai BN, Mullin GE, Limsui D, Parian AM. Role of vitamin D in inflammatory bowel disease. Nutrition in Clinical Practice. 2017; 32(3): 337-345.

[25] Munger KL, Levin LI, Hollis BW, Howard NS, Ascherio A. Serum 25hydroxyvitamin D levels and risk of multiple sclerosis. Jama. 2006; 296(23): 2832-2838.

[26] Zeitelhofer M, Adzemovic MZ, Gomez-Cabrero D, Bergman P, Hochmeister S, N'diaye M, et al. Functional genomics analysis of vitamin $\mathrm{D}$ effects on $\mathrm{CD} 4+\mathrm{T}$ cells in vivo in experimental autoimmune encephalomyelitis. Proceedings of the National Academy of Sciences. 2017; 114(9): E1678-87.

[27] Del Valle DM, Kim-Schulze S, Huang HH, Beckmann ND, Nirenberg $\mathrm{S}$, Wang B, et al. An inflammatory cytokine signature predicts COVID19 severity and survival. Nature Medicine. 2020; 26(10): 1636-1643.

[28] Henry BM, De Oliveira MH, Benoit S, Plebani M, Lippi G. Hematologic, biochemical and immune biomarker abnormalities associated with severe illness and mortality in coronavirus disease 2019 (COVID-19): a meta-analysis. Clinical Chemistry and Laboratory Medicine (CCLM). 2020; 58(7): 1021-1028.

[29] Wright DJ. Prevention of the cytokine storm in COVID-19. The Lancet Infectious Diseases. 2021; 21(1): 25-26.

[30] Besnier E, Tuech JJ, Schwarz L. We asked the experts: Covid-19 outbreak: is there still a place for scheduled surgery?"Reflection from pathophysiological data". World Journal of Surgery. 2020; 44(6): 1695-1698. 
[31] Ulhaq ZS, Soraya GV. Interleukin-6 as a potential biomarker of COVID-19 progression. Medecine et Maladies Infectieuses. 2020; 50(4): 382.

[32] Scherger S, Henao-Martínez A, Franco-Paredes C, Shapiro L. Rethinking interleukin-6 blockade for treatment of COVID-19. Medical Hypotheses. 2020; 144: 110053.

[33] Coomes EA, Haghbayan H. Interleukin-6 in COVID-19: a systematic review and meta-analysis. Reviews in Medical Virology. 2020; 30(6): $1-9$.

[34] Leija-Martínez JJ, Huang F, Del-Río-Navarro BE, Sanchéz-Muñoz F, Muñoz-Hernández O, Giacoman-Martínez A, et al. IL-17A and TNFas potential biomarkers for acute respiratory distress syndrome and mortality in patients with obesity and COVID-19. Medical Hypotheses. 2020; 144: 109935.

[35] Perlin DS, Zafir-Lavie I, Roadcap L, Raines S, Ware CF, Neil GA. Levels of the TNF-related cytokine light increase in hospitalized COVID-19 patients with cytokine release syndrome and ARDS. MSphere. 2020; 5(4): e00699-e00720.

[36] Tjan LH, Furukawa K, Nagano T, Kiriu T, Nishimura M, Arii J, et al. Early Differences in Cytokine Production by Severity of Coronavirus Disease 2019. The Journal of Infectious Diseases. 2021; 223(7): 11451149.

[37] Huang CG, Dutta A, Huang CT, Chang PY, Hsiao MJ, Hsieh YC. Relative COVID-19 viral persistence and antibody kinetics. Pathogens. 2021; 10(6): 752.

[38] Guo J, Wang S, Xia H, Shi D, Chen Y, Zheng S, et al. Cytokine Signature Associated with Disease Severity in COVID-19. Frontiers in Immunology. 2021: 3276.

[39] Kalia V, Xiao H, Yuzefpolskiy Y, Sarkar S. IL-2 Signals Program the Fate of Exhausted CD8 T cells, 2019.

[40] Del Valle DM, Kim-Schulze S, Huang HH, Beckmann ND, Nirenberg $\mathrm{S}$, Wang B, et al. An inflammatory cytokine signature predicts COVID19 severity and survival. Nature Medicine. 2020; 26(10): 1636-1643. 